\title{
Surgical treatment of parapharyngeal space tumors: A report of 29 cases
}

\author{
KEI IJICHI and SHINGO MURAKAMI \\ Department of Otolaryngology-Head and Neck Surgery, Nagoya City University Graduate School of Medical Sciences, \\ Nagoya, Aichi 467-8601, Japan
}

Received March 12, 2017; Accepted May 16, 2017

DOI: $10.3892 / \mathrm{ol} .2017 .6480$

\begin{abstract}
The present study aimed to establish a strategy for parapharyngeal space (PPS) tumor surgery based on preoperative symptoms, clinical signs, imaging and histological examination. A retrospective cohort study was conducted with 29 adult patients who underwent surgery for primary PPS tumors from 2008-2015. The following data was obtained: Preoperative symptoms of the patient, histological type of the tumor, surgical approach and complications. Of the 29 patients who underwent surgery to remove a PPS tumor, 16 presented with neurogenic tumors and 13 with salivary gland tumors. The most common symptom was the presence of a neck mass. Preoperative computed tomography and magnetic resonance imaging were performed to evaluate the size and location of the tumors. The majority of the salivary gland tumors were located in the prestyloid space, whereas all neurogenic tumor cases were poststyloid. A total of 21 of the 29 patients $(72.4 \%)$ underwent a preoperative fine needle aspiration (FNAC) examination. FNAC contributed to preoperative diagnosis in 9 of these cases (42.9\%). In parotid tumors of the prestyloid space, facial nerve palsy was a common postoperative complication. In the case of neurogenic tumors, a common complication was the postoperative palsy of the nerve of tumor origin. The strategy for PPS surgery, from the preoperative diagnosis to the operative method, remains controversial. The data on pre- and postoperative symptoms, imaging and histological diagnosis, and the selection of surgical method depending on a neurogenic or salivary tumor origin in the present study indicated that improving the method of examination, carefully selecting the method of approach and accurately managing surgery leads to complete tumor removal, and that the use
\end{abstract}

Correspondence to: Dr Kei Ijichi, Department of OtolaryngologyHead and Neck Surgery, Nagoya City University Graduate School of Medical Sciences, 1 Kawasumi, Mizuho-cho, Mizuho-ku, Nagoya, Aichi 467-8601, Japan

E-mail: ijichi@med.nagoya-cu.ac.jp

Key words: parapharyngeal space, neck tumor, schwannoma, salivary gland tumor of nerve-preserving techniques may reduce the likelihood of complications.

\section{Introduction}

The parapharyngeal space (PPS) is located in the suprahyoid neck between the hyoid bone and the skull base, alongside the pharynx. It is divided into prestyloid and poststyloid compartments, separated by the tensor veli palatini muscle and styloid process $(1,2)$. PPS tumors are rare and account for $\sim 0.5 \%$ of head and neck tumors (3). Of these tumors, $\sim 80 \%$ are benign (4). The most frequent PPS tumor is a salivary neoplasm, followed by a neurogenic tumor (5-8). Salivary neoplasms in the PPS, particularly pleomorphic adenomas, are the most common lesions in the prestyloid space, whereas paragangliomas and schwannomas are the most common in the poststyloid space (5). Malignancy is also most common in tumors originating in the salivary glands (9).

Pre-operative diagnosis of the PPS tumor assists the determination of a surgical plan. Computed tomography (CT) and magnetic resonance imaging (MRI) scans are crucial for the assessment of PPS tumors. MRI is particularly useful for determining the tumor localization and distinguishing the tumor origin. Assignment of the tumor to the prestyloid or poststyloid compartment depending on its localization provides a potential diagnosis (1,10-12). If a paraganglioma is suspected, in which the tumor is located around the bifurcation of the carotid artery, an angiography or CT angiography is recommended $(6,7,10)$.

Surgery is the mainstay of treatment for PPS tumors, and is performed on the basis of information provided by these diagnostic modalities. A complex structure consisting of major blood vessels, the cranial nerves (CNs), several multidirectional muscles, and the jawbone and cranial bones cause the PPS to be narrow and difficult to approach $(10,13,14)$.

There are several surgical approaches that it is possible to use for the resection of PPS tumors. These are predominantly classified into four groups: Transcervical, transparotid, transoral and transmandibular approaches. These approaches, or a combined approach, are performed in the surgery of PPS tumors depending on the characteristics of the tumor $(2,10,12,15)$.

In the present study, the diagnosis, surgical approach and complications associated with surgical PPS tumor treatment in our experience are reported on, and previous studies are discussed, to aid in improving the outcome of this disease. 


\section{Materials and methods}

The present, retrospective study included 29 patients who underwent surgery for primary PPS tumors between January 2008 and December 2015 in Nagoya City University Hospital (Nagoya, Japan). The protocol of the investigation was approved by the Institutional Review Board of Nagoya City University Hospital (Nagoya, Japan). Informed consent was obtained from the patients prior to their inclusion in the study.

In regard to histologic type, there were 16 cases of neurogenic and 13 of salivary gland origin tumors. The following data were evaluated: Preoperative symptoms, histological type, surgical approach and complications Patients were evaluated following a laboratory examination. Enhanced CT and MRI scans were used to confirm the location and size of the tumor as a preoperative diagnosis. In particular, the following aspects were evaluated: Tumor shape and the tumor margin, the association of the tumor location with major vasculature, surrounding tissue and the deep lobe of the parotid gland (DLPG), and whether the tumor localization was pre- or poststyloid. Preoperative imaging was also used to estimate the origin of the tumor, the extent of malignancy and tumor vascularity. For the examination of the histology of the tumor, fine needle aspiration cytology (FNAC) was performed in the majority of cases. Fine needle aspiration biopsy (FNAB) or incisional biopsy were required in certain cases when the histological results were unclear and there was a suspicion of malignancy. In the case of malignancy, additional imaging examination was performed to inform the selection of a treatment plan. Where tumors exhibited the possibility of paraganglioma, angiography or CT-angiography was performed to evaluate the vasculature associated with the carotid artery.

All patients enrolled in the study underwent surgical treatment. The plan for the surgical approach was selected according to the tumor location, histological findings, the relationship to anatomical structures and the suspicion of malignancy. The approach of wide resection and reconstruction was selected in cases where malignancy was confirmed. During the follow-up period, clinical examination was performed each month following surgical resection and an MRI of the head and neck was performed at annual intervals for 5 years. Patients with malignant cases underwent a head and neck CT or MRI every 6 months during the first year, and head, neck, lung and abdominal CT or MRI scans annually. If patients exhibited abnormal symptoms (e.g. neck mass or lower cranial nerve palsy) during the follow-up, an additional examination was performed, in case of recurrence.

\section{Results}

Preoperative symptoms. There were 11 males and 18 females included in the present study. The mean age at the point of surgery was $43.5 \pm 16.3$ years (range, $19-73$ years) and the mean follow-up duration was $29.3 \pm 25.4$ months (range, 1.8-88.1 months). Pathological diagnosis resulted in 16 cases being classified as a neurogenic tumor (55.2\%) and 13 cases as a salivary gland tumor (44.8\%; Table I). The most common symptoms of neurogenic tumors were a neck mass (37.5\%), coughing (12.5\%), hoarseness (12.5\%) and pharyngeal pain (12.5\%; Table IIA). The most common symptoms of salivary gland tumors were the presence of a mass on the neck (30.8\%) and abnormal sensation of the pharynx (15.4\%; Table IIB).

Imaging examination. Preoperative CT or MRI imaging was used to evaluate the location of each tumor. A total of 9 salivary gland tumors were located in the prestyloid space and 3 cases were located in the pre- and poststyloid spaces. The remaining case, a malignant parotid tumor, was difficult to judge as it was localized between the prestyloid and poststyloid spaces. All 16 schwannoma cases were in the poststyloid space (Table I). The size of each tumor was measured with CT or MRI imaging. For neurogenic tumors, the most frequent range for tumor size was $30-50 \mathrm{~mm}$ (7 cases; $43.8 \%)$ and the most frequent range for salivary tumor size was $40-50 \mathrm{~mm}$ (5 cases; $38.5 \%)$.

Histopathologic examination. A total of 21 of the 29 patients (72.4\%) underwent a preoperative FNAC and 6 patients (27.3\%) underwent a preoperative histopathological evaluation. FNAB was performed in 2 cases (6.9\%) to confirm prestyloid schwannoma following FNAC results from which there were suspicions of malignancy. The cytological examination revealed pleomorphic adenoma in 11 cases (84.6\%), including cellular atypia in 4 cases (36.4\%) and lymphocytes in 1 case $(9.1 \%)$. Of the 10 neurogenic tumors for which it was performed, FNAC revealed spindle cells in 3 cases (30.0\%) and unclear results in 7 cases $(70.0 \%)$. FNAC examination only contributed to preoperative diagnosis in 9 cases (42.9\%), whereas FNAB examination contributed to the surgical plan in the 2 cases where it was performed.

Surgical method. Tumor resection was performed in all cases. For all patients with a benign tumor, the transcervical, transparotid or combined approach was selected. In patients with a malignant tumor, the mandibular swing approach was used to resect the tissues surrounding the tumor. The tumor was then exposed through the space between the submandibular gland and parotid gland. If approaching the tumor was difficult, the digastric post-berry and stylo-hyoid muscle was cut. A microscope was used to improve the visibility of the area surrounding the tumor.

In cases of neurogenic tumors, the transcervical approach was used. A nerve integrity monitor was used for lower $\mathrm{CN}$ monitoring, including for the VII, IX, X, XI and XII nerves. In schwannoma cases, tumor enucleation was performed to preserve nerve functionality. In paraganglioma and neurofibroma cases, an en bloc resection of the tumor was performed. In tumors of salivary gland origin, the transcervical or transparotid approach was selected, according to tumor location. Tumors were performed with en bloc resection, avoiding tumor spillage. In the single case of a malignant salivary gland tumor, the mandibular swing approach was performed and the tumor was resected with a wide surgical margin as the tumor had invaded the surrounding tissue.

Postoperative complications. Major complications following surgery are summarized in Table III. In salivary origin tumors of the prestyloid space, facial nerve palsy was a complication 
Table I. Characteristics of patients according to tumor origin.

A, Characteristics of patients with neurogenic tumors, $n=16$

\begin{tabular}{|c|c|c|c|c|c|}
\hline Age & Sex & Approach & Tumor location & Final pathological diagnosis & Original nerve \\
\hline 23 & $\mathrm{~F}$ & Cervical & Poststyloid & Schwannoma & Sympathetic nerve \\
\hline 28 & M & Cervical & Poststyloid & Schwannoma & Sympathetic nerve \\
\hline 19 & $\mathrm{~F}$ & Cervical & Poststyloid & Schwannoma & Sympathetic nerve \\
\hline 38 & $\mathrm{~F}$ & Cervical & Poststyloid & Schwannoma & Sympathetic nerve \\
\hline 38 & $\mathrm{~F}$ & Cervical & Poststyloid & Schwannoma & Sympathetic nerve \\
\hline 19 & M & Cervical & Poststyloid & Schwannoma & Sympathetic nerve \\
\hline 67 & M & Cervical & Poststyloid & Schwannoma & Sympathetic nerve \\
\hline 64 & M & Cervical & Poststyloid & Schwannoma & Hypoglossus nerve \\
\hline 28 & $\mathrm{~F}$ & Cervical & Poststyloid & Paraganglioma & Carotid body \\
\hline 45 & $\mathrm{M}$ & Cervical & Poststyloid & Schwannoma & Hypoglossus nerve \\
\hline 44 & $\mathrm{~F}$ & Cervical & Poststyloid & Schwannoma & Hypoglossus nerve \\
\hline 37 & $\mathrm{~F}$ & Cervical & Poststyloid & Neurofibroma & Sympathetic nerve \\
\hline 33 & $\mathrm{M}$ & Cervical & Poststyloid & Schwannoma & Vagus nerve \\
\hline 34 & $\mathrm{M}$ & Cervical & Poststyloid & Schwannoma & Vagus nerve \\
\hline 35 & $\mathrm{M}$ & Cervical & Poststyloid & Schwannoma & Vagus nerve \\
\hline 36 & M & Cervical & Poststyloid & Schwannoma & Vagus nerve \\
\hline
\end{tabular}

$\mathrm{B}$, Characteristics of patients with salivary gland tumors, $\mathrm{n}=13$

\begin{tabular}{|c|c|c|c|c|}
\hline Age & Sex & Approach & Tumor location & Final pathological diagnosis \\
\hline 47 & $\mathrm{~F}$ & Cervical & Prestyloid & Pleomorphic adenoma \\
\hline 61 & M & Cervical & Prestyloid & Pleomorphic adenoma \\
\hline 49 & $\mathrm{~F}$ & Cervical & Prestyloid & Pleomorphic adenoma \\
\hline 64 & $\mathrm{~F}$ & Cervical & Prestyloid-poststyloid & Pleomorphic adenoma \\
\hline 54 & $\mathrm{~F}$ & Parotid-cervical & Prestyloid-poststyloid & Pleomorphic adenoma \\
\hline 73 & M & Cervical & Prestyloid & Pleomorphic adenoma \\
\hline 66 & M & Cervical & Prestyloid-poststyloid & Pleomorphic adenoma \\
\hline 30 & M & Parotid-cervical & Prestyloid & Pleomorphic adenoma \\
\hline 54 & $\mathrm{~F}$ & Cervical & Prestyloid & Pleomorphic adenoma \\
\hline 53 & M & Cervical & Prestyloid & Pleomorphic adenoma \\
\hline 48 & $\mathrm{~F}$ & Transmandibular & Poststyloid & Carcinoma in pleomorphic adenoma \\
\hline 25 & $\mathrm{~F}$ & Parotid-cervical & Prestyloid & Pleomorphic adenoma \\
\hline 33 & M & Parotid-cervical & Prestyloid & Carcinoma in pleomorphic adenoma \\
\hline
\end{tabular}

observed in 5 cases (38.5\%). The most common location for facial palsy was under the lip (4 cases). Total facial nerve palsy occurred in 2 cases, although 1 of these patients, who had a benign tumor, recovered after 4 months. Frey syndrome was not observed in any case. First bite syndrome (FBS) occurred in 5 cases $(38.5 \%)$ and was resolved in 3 cases $(60.0 \%)$. FBS continues in 2 cases $(40.0 \%)$, was resolved within 1 year in 2 cases $(40.0 \%)$ and was resolved within 1 to 2 years in 1 case $(20.0 \%)$. In the case of neurogenic tumors, the tumor was resected with intracapsular enuclation in order to preserve the original nerve. A total of 10 patients with neurogenic tumors exhibited palsy following surgery. The paralysis was resolved in $12-18$ months for 4 patients $(40.0 \%)$, whereas the remaining patients $(60.0 \%)$ continue to exhibit paralysis (Table IV). FBS occurred in 5 cases $(31.3 \%)$, continues in 4 cases $(80.0 \%)$, and was resolved within 1 year in 1 case $(20.0 \%)$. In a case of paraganglioma, the patient had transient XII nerve palsy. It was resolved after 3 months. Tumor recurrence was observed in 1 plexiform schwannoma case following resection. Surgery was performed a short time following the diagnosis of tumor recurrence. In malignant tumor cases, VII nerve palsy occurred due to the necessary resection of the nerve. Facial nerve mandibular branch (FNMB) palsy was also exhibited in one case where surgery was performed on the lower cheek flap. No patients presented with free flap issues following the cheek surgery. 
Table II. Incidence of preoperative symptoms in parapharyngeal space tumors.

A, Preoperative symptoms of neurogenic tumors, $n=16$

\begin{tabular}{ll}
\hline Symptoms & $\mathrm{n}(\%)$ \\
\hline Neck mass & $6(37.5)$ \\
Cough & $2(12.5)$ \\
Hoarseness & $2(12.5)$ \\
Abnormal sensation of the pharynx & $1(6.3)$ \\
Tongue palsy & $1(6.3)$ \\
Sleep apnea & $1(6.3)$ \\
No symptoms & $1(6.3)$ \\
\hline
\end{tabular}

B, Preoperative symptoms of salivary gland tumors, $\mathrm{n}=13$

Neck mass

$4(30.8)$

No symptoms

Abnormal sensation of the pharynx

Pharyngeal pain

Pharyngeal mass

Posterior neck pain

$4(30.8)$

$2(15.4)$

$1(7.7)$

$1(7.7)$

$1(7.7)$

Table III. Incidence of postoperative complications in salivary gland tumors.

\begin{tabular}{ll}
\hline Symptoms & $\mathrm{n}(\%)$ \\
\hline First bite syndrome & $6(46.2)$ \\
Lower lip palsy & $4(30.8)$ \\
Total facial nerve palsy & $1(7.7)$ \\
Abnormal feeling of neck & $1(7.7)$ \\
Pharyngeal pain & $1(7.7)$ \\
\hline
\end{tabular}

\section{Discussion}

PPS tumors occur deep within the neck, and this location results in difficulties in diagnosis and surgical treatment. The purpose of the present study was to evaluate the PPS treatment process and characteristics from diagnosis to surgical treatment and thereafter. There was also the opportunity to evaluate the utility of functional preservation surgery with lower $\mathrm{CN}$ monitoring. According to previous reports, 70-90\% of PPS tumors are benign $(6,7,9-11,14,16,17)$. Only 2 cases $(8 \%)$ of the cohort examined in the present study were malignant, which was less than in these other studies. Previous studies have reported that the most common subtype is pleomorphic adenoma originating from the deep lobe of the salivary gland $(4,16)$, whereas Carrau et al (6) reported that $57 \%$ of PPS neoplasms were neurogenic tumors. In the present study, $>50 \%$ of the cases were schwannoma. The most frequent origin nerves of the schwannomas were the sympathetic and vagus nerves. Liu et al (18) corroborated this observation, reporting that the sympathetic and vagus nerves were the most common nerves of origin in head and neck schwannomas (18). However, no previous study has described the nerves of origin of PPS schwannomas. A study by Tryggvason et al (19) revealed that schwannomas arise in nerves with a sensory component and are associated with sensory ganglia. In addition, it was reported that the majority of sympathetic chain schwannomas are associated with the superior cervical ganglion (19). It is thus logical that these nerves are common origins for PPS schwannomas.

The most frequent symptom in the cohort of the present study was a neck mass, followed by pharyngeal mass; this is comparable with other studies $(6,15)$. Dysphasia and pain have also been reported to be common symptoms $(6,10,15)$. No patients presented with these symptoms in the present study. A total of 2 patients with neurogenic tumors exhibited preoperative neural deficits in the present study. The tumor mass resulted in a deficit caused by injury to the nerve of origin (XII) in 1 patient, and in the remaining case, the symptoms were due to injury of the adjacent nerve (X) from a tumor of the XII nerve. In particular, the vagus nerve contacts with the sublingual nerve. A previous study has reported that, for malignant tumors, the most frequent symptoms are a rapidly growing neck mass, pain, trismus, otalgia and cranial nerve deficits (2). A malignant tumor in the present study presented with the symptom of a neck mass.

Preoperative imaging analysis of the PPS is performed with the aim of providing information as follows: i) Size of tumor; ii) location of tumor (pre- or poststyloid, relation to parotid gland) and iii) whether there is extension of the tumor to the adjacent area and major vasculature. MRI imaging is often able to provide this information for soft tissue. A previous study reported the superiority of MRI vs. CT as it permits the improved discrimination of soft tissue types (20). Enhanced $\mathrm{CT}$ is useful for analysis of tumor location and to preoperatively establish a method of approach for surgery (10). Three dimensional-CT angiography is indicated when the tumor is suspected to be a carotid body tumor from its location, in order to evaluate its vascularity and confirm the diagnosis (21).

It is also necessary to distinguish between benign and malignant tumors prior to surgery. In a previous study, CN palsy and pain were identified as symptoms likely to be associated with malignancy (2). However, in the present study, just one patient with PPS schwannomas exhibited preoperative $\mathrm{CN}$ palsy; therefore, $\mathrm{CN}$ palsy is not a specific symptom for malignant PPS tumors. Previous reports have stated that the radiological signs of malignancy include irregular tumor margins, spread into surrounding tissues and fat planes as determined by CT or MRI, and evidence of enlarged, necrotic lymph nodes in the retropharyngeal and cervical area $(2,8,10)$.

Histological evaluation, particularly when there are suspicions of malignancy, is desirable. Performing an incisional biopsy carries the risk of tumor dissemination; however, it is suggested when there is suspicion of a malignant neoplasm (6). The PPS is an unusual target for FNAB and is associated with certain disadvantages, including difficulty of access (2). FNAC of PSS tumors is performed for lesions of the prestyloid PPS and may allow for the differentiation of salivary gland malignancies. Needle biopsy of the poststyloid PPS carries the risk of vascular complications (22), and its use is also limited in cases of the diagnosis of hemorrhagic specimens in paragangliomas and hypocellular specimens in schwannomas $(23,24)$. 
Table IV. Incidence of nerve deficiency as a postoperative symptom, according to the original nerve.

\begin{tabular}{|c|c|c|c|}
\hline Origin & Symptom of nerve deficiency & Frequency, n (\%) & Symptom continuation, n (\%) \\
\hline \multirow[t]{2}{*}{ Synpathetic nerve, $n=10$} & Horner syndrome & $5(50.0)$ & $4(40.0)$ \\
\hline & Tongue palsy & $1(10.0)$ & $1(10.0)$ \\
\hline \multirow{3}{*}{ Vagus nerve, $n=4$} & Vocal cord palsy & $2(50.0)$ & $0(0.0)$ \\
\hline & Tongue palsy & $2(50.0)$ & $1(25.0)$ \\
\hline & Pharyngoparalysis & $2(50.0)$ & $1(25.0)$ \\
\hline \multirow[t]{2}{*}{ Hypoglossal nerve, $n=3$} & Tongue palsy & $3(100.0)$ & $2(66.7)$ \\
\hline & Vocal cord palsy & $2(66.7)$ & $2(33.3)$ \\
\hline \multirow[t]{2}{*}{ Carotid body tumor, $n=1$} & Tongue palsy & $1(100.0)$ & $0(0.0)$ \\
\hline & Pharyngoparalysis & $1(100.0)$ & $0(0.0)$ \\
\hline
\end{tabular}

\section{Preoperative examination for PPS tumor surgery}

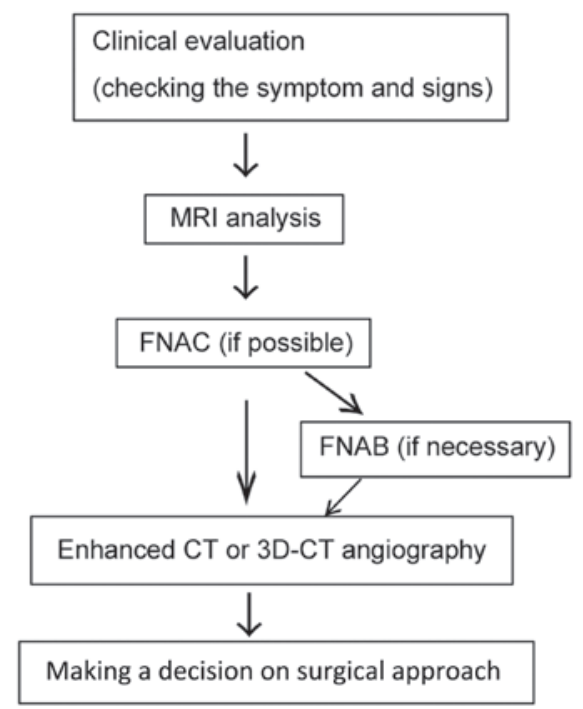

Figure 1. Flowchart depicting the preoperative examination of PPS tumors.

A flow chart of the complete preoperative examination process is presented in Fig. 1.

A number of authors have described the indications for a transcervical, transoral, transparotid-transcervical or transmandibular approach to surgery. The transcervical approach is most frequently used to access PPS tumors $(6,7,15)$, and was performed in the present study for all neurogenic tumors and $61.5 \%$ of salivary gland tumors (Table I). This approach allows direct access to the PPS. The skin is incised from below the submandibular gland to below the parotid gland. At the deeper layer, it is possible to treat the digastric, stylohyoid and stylomandibular ligaments (10). If the vertical extension of the tumor causes suspicions of intracranial extension, it may be difficult to use a transcervical approach to dissect the tumor from the surrounding tissues, particularly when it is in the vicinity of the cranial base (2). In cases of malignant tumors, difficulties in obtaining clear margins may arise from the restricted visibility of this approach (15). The transparotid-transcervical approach is suitable for prestyloid tumors arising in the DLPG that extend into the PPS, requiring the identification and preservation of each branch of the facial nerves (10). The transoral approach presents a direct route to tumors though the oropharynx, but provides no control of the large blood vessels (6). This approach may be useful for small benign avascular tumors that present in the oropharynx and do not extend to the styloid process $(14,19,25,26)$. However, other authors have reported that this approach for PPS tumor was associated with a $25 \%$ recurrence rate within 5 years (27). Furthermore, a number of authors avoid using this approach due to the risk of damaging the blood vessels and the potential for incomplete tumor extirpation $(6,7,10)$. Robotic transoral surgery of the PPS tumor was previously reviewed, and the authors determined that the risk of violation of the capsule of a pleomorphic adenoma was most severe when the capsule was ruptured and tumor spillage occurred (28). In our opinion, this would produce an unacceptable risk to prognosis; tumor localization, size and suspicions of malignancy should be carefully reviewed prior to considering this approach. In the present study, the indications for the transmandibular approach in PPS surgery were malignant neoplasms. Large recurrent neoplasms, large benign neoplasms and highly vascular neoplasms where improved vascular control is required also indicate this approach $(9,29)$. This approach allows the safe resection of lesions invading the skull base that need further exposure (7). The use of infratemporal fossa approaches, including the orbito-zygomatic-middle fossa approach, is limited to PPS tumors surrounding the temporal, lateral skull base and the infratemporal fossa. This approach is used in cases of trigeminal schwannoma (30).

Tracheostomy is not ordinarily necessary for the surgery of a benign PPS tumor. If a defect following tumor resection requires reconstruction with a musculocutanous flap that may cause narrowing of the upper respiratory tract, tracheostomy is performed at the end of the surgery, as previously reported (10). Particular cases where tracheostomy was indicated in a previous study included cases of tumors $>10 \mathrm{~cm}$, or cerebral infarction (2).

In previous studies, the most frequently reported complication of PPS tumor surgery was FNMB palsy $(10,15)$. These studies predominantly enrolled patients with a pleomorphic adenoma of the PPS. In the cohort of the present study, FNMB palsy typically occurred following surgery to remove tumors of salivary origin, as the surgery was performed close to the mandible. The second most common complication in these previous studies was the presentation of FBS. FBS also occurred in the present study, following prestyloid tumor 
surgery. Frey syndrome was reported only in the deep lobe of tumors treated with the transcervical-transparotid approach, but the cause was not described in these previous reports.

The most serious complication of PPS tumor resection is CN paralysis. The associated CNs are VII, IX, X, XI and XII (31). The original nerve of the tumor tends to be injured following the resection of neurogenic tumors, including schwannomas and paragangliomas. $\mathrm{CN} X$ palsy is the most common form (32), and may be severe following PPS surgery. A previous study (4) revealed that neurogenic tumors, particularly vagal paragangliomas, are particularly at risk for developing postoperative sequelae. Horner syndrome may also be presented postoperatively in cases of sympathetic PPS schwannomas (4). The tumor enucleation of PPS schwannomas may allow the preservation of the function of the original nerves. Mapping the nerve fiber with an electromyographic (EMG) system may help to decide the incision line on the tumor capsule $(33,34)$. In the present study, an EMG system was used when there was suspicion of a neurogenic tumor, particularly schwannomas, in order to lessen damage to the nerve fibers as much as possible during tumor enucleation. When a nerve bundle is visible, the tumor should be incised where it is separated from the nerve fiber. When the nerve bundle is not visible, the tumor should be incised where it swells $(33,34)$. An EMG system is particularly useful to map the nerve fibers of motor nerves.

The strategy used during PPS surgery should be dependent on the status of the tumor. In the present study, this was determined with information obtained via clinical evaluation, imaging analysis and histological analysis. The most common histologic types were schwannoma or pleomorphic adenoma. In schwannoma cases, tumors should be enucleated to preserve the function of the lower CNs. When pleomorphic adenomas are resected, tumor spillage should be avoided to prevent relapse.

The major goals of PPS tumor resection are to completely remove the tumor and reduce the number of postoperative complications. This may be achieved by improving the methods of examination, carefully selecting the approach for surgery and with accurate management during surgery.

\section{References}

1. Curtin HD: Separation of the masticator space from the parapharyngeal space. Radiology 163: 195-204, 1987

2. Basaran B, Polat B, Unsaler S, Ulusan M, Aslan I and Hafiz G: Parapharyngeal space tumours: The efficiency of a transcervical approach without mandibulotomy through review of 44 cases. Acta Otorhinolaryngol Ital 34: 310-316, 2014.

3. Stell PM, Mansfield AO and Stoney PJ: Surgical approaches to tumors of the parapharyngeal space. Am J Otolaryngol 6: 92-97, 1985.

4. Cohen SM, Burkey BB and Netterville JL: Surgical management of parapharyngeal space masses. Head Neck 27: 669-675, 2005.

5. Batsakis JG and Sneige N: Parapharyngeal and retropharyngeal space diseases. Ann Otol Rhinol Laryngol 98: 320-321, 1989.

6. Carrau RL, Myers EN and Johnson JT: Management of tumors arising in the parapharyngeal space. Laryngoscope 100: 583-589, 1990.

7. Khafif A, Segev Y, Kaplan DM, Gil Z and Fliss DM: Surgical management of parapharyngeal space tumors: A 10-year review. Otolaryngol Head Neck Surg.132: 401-406, 2005.

8. Work WP and Hybels RL: A study of tumors of the parapharyngeal space. Laryngoscope.84: 1748-1755, 1974.

9. Shahab R, Heliwell T and Jones AS: How we do it: A series of 114 primary pharyngeal space neoplasms. Clin Otolaryngol 30: $364-367,2005$
10. Dimitrijevic MV, Jesic SD, Mikic AA, Arsovic NA and Tomanovic NR: Parapharyngeal space tumors: 61 case reviews. Int J Oral Maxillofac Surg 39: 983-989, 2010.

11. Allison RS, Van der Waal I and Snow GB: Parapharyngeal tumours: A review of 23 cases. Clin Otolaryngol Allied Sci 14: 199-203, 1989.

12. Kuet ML, Kasbekar AV, Masterson L and Jani P: Management of tumors arising from the parapharyngeal space: A systematic review of 1,293 cases reported over 25 years. Laryngoscope 125: 1372-1381, 2015.

13. Horowitz G, Ben-Ari O, Wasserzug O, Weizman N, Yehuda M and Fliss DM: The transcervical approach for parapharyngeal space pleomorphic adenomas: Indications and technique. PLoS One 9: e90210, 2014.

14. Olsen KD: Tumors and surgery of the parapharyngeal space. Laryngoscope 104 (5 Pt 2 Suppl 63): S1-S28, 1994.

15. Papadogeorgakis N, Petsinis V, Goutzanis L, Kostakis G and Alexandridis C: Parapharyngeal space tumors: Surgical approaches in a series of 13 cases. Int J Oral Maxillofac Surg 39: 243-250, 2010.

16. Eneroth CM: Histological and clinical aspects of parotid tumours. Acta Otolaryngol Suppl 188 (Suppl 191): S1-S99, 1964.

17. Hughes KV III, Olsen KD and McCaffrey TV: Parapharyngeal space neoplasms. Head Neck 17: 124-130, 1995.

18. Liu HL, Yu SY, Li GK and Wei WI: Extracranial head and neck Schwannomas: A study of the nerve of origin. Eur Arch Otorhinolaryngol 268: 1343-1347, 2011.

19. Tryggvason G, Barnett A, Kim J, Soken H, Maley J and Hansen MR: Radiographic association of schwannomas with sensory ganglia. Otol Neurotol 33: 1276-1282, 2012.

20. Som PM, Sacher M, Stollman AL, Biller HF and Lawson W: Common tumors of the parapharyngeal space: Refined imaging diagnosis. Radiology 169: 81-85, 1988.

21. Caldarelli C, Bucolo S, Spisni R and Destito D: Primary parapharyngeal tumours: A review of 21 cases. Oral Maxillofac Surg 18: 283-292, 2014.

22. Arnason T, Hart RD, Taylor SM, Trites JR, Nasser JG and Bullock MJ: Diagnostic accuracy and safety of fine-needle aspiration biopsy of the parapharyngeal space. Diagn Cytopathol 40: 118-123, 2012.

23. Zaharopoulos P: Diagnostic challenges in the fine-needle aspiration diagnosis of carotid body paragangliomas: Report of two cases. Diagn Cytopathol 23: 202-207, 2000.

24. Yu GH, Sack MJ, Baloch Z and Gupta PK: Difficulties in the fine needle aspiration (FNA) diagnosis of schwannoma. Cytopathology 10: 186-194, 1999.

25. Bass RM: Approaches to the diagnosis and treatment of tumors of the parapharyngeal space. Head Neck Surg 4: 281-289, 1982.

26. Luna-Ortiz K, Navarrete-Alemán JE, Granados-García M and Herrera-Gómez A: Primary parapharyngeal space tumors in a Mexican cancer center. Otolaryngol Head Neck Surg 132: $587-591,2005$.

27. Goodwin WJ Jr and Chandler JR: Transoral excision of lateral parapharyngeal space tumors presenting intraorally. Laryngoscope 98: 266-269, 1988.

28. Chan JY, Tsang RK, Eisele DW and Richmon JD. Transoral robotic surgery of the parapharyngeal space: A case series and systematic review. Head Neck 37: 293-298, 2015.

29. Kolokythas A, Eisele DW, El-Sayed I and Schmidt BL: Mandibular osteotomies for access to select parapharyngeal space neoplasms. Head Neck 31: 102-110, 2009.

30. Krishnamurthy S, Holmes B and Power SK: Schwannomas limited to the infratemporal fossa: Report of two cases. J Neurooncol 36: 269-277, 1998.

31. Presutti L, Molteni G, Malvè L, Marchioni D, Ghidini A, Tassi S, Chiarini L and Alicandri-Ciufelli M: Parapharyngeal space tumors without mandibulotomy: Our experience. Eur Arch Otorhinolaryngol 269: 265-273, 2012.

32. Yasumatsu R, Nakashima T, Miyazaki R, Segawa Y and Komune S: Diagnosis and management of extracranial head and neck schwannomas: A review of 27 cases. Int J Otolaryngol 2013: 973045, 2013.

33. Behuria S, Rout TK and Pattanayak S: Diagnosis and management of schwannomas originating from the cervical vagus nerve. Ann R Coll Surg Engl 97: 92-97, 2015.

34. Boufettal M, Azouz M, Rhanim A, Abouzahir M, Mahfoud M, Bardouni AE, Berrada MS and Yaacoubi ME: Schwannoma of the median nerve: Diagnosis sometimes delayed. Clin Med Insights Case Rep 7: 71-73, 2014. 